\title{
The Modeling of Redundantly-actuated Mechanical Systems
}

\author{
Yaojun Wang \\ Faculty of Mechanical Engineering and Automation \\ Zhejiang Sci-Tech University \\ Hangzhou, Zhejiang 310018, China \\ Department of Electrical and Electronic Engineering \\ Zhejiang Institute of Mechanical and Electrical Engineering \\ Hangzhou, Zhejiang 310053, China \\ Email: yaojunw@vip.163.com \\ Bruno Belzile \\ Department of Mechanical Engineering \\ and Centre for Intelligent Machines \\ McGill University \\ Montreal, QC H3A 0C3, Canada \\ Email: bruno@cim.mcgill.ca \\ Jorge Angeles \\ Department of Mechanical Engineering \\ and Centre for Intelligent Machines \\ McGill University \\ Montreal, QC H3A 0C3, Canada \\ Email: angeles@cim.mcgill.ca \\ Qinchuan Li* \\ Faculty of Mechanical Engineering and Automation \\ Zhejiang Sci-Tech University \\ Hangzhou, Zhejiang 310018, China \\ Email: Iqchuan@zstu.edu.cn
}

\begin{abstract}
Dynamics modeling is essential in the design and control of mechanical systems, the focus of the paper being redundantly-actuated systems, which bring about special challenges. The authors resort to the natural orthogonal complement (NOC), based on an adaptation of screw theory, to derive the dynamics model. Benefiting from the elimination of the constraint wrenches, the NOC offers a simple, systematic alternative to the modeling of redundantly-actuated mechanical systems. The optimum actuator-torque distribution is determined via Euclideannorm minimization; then, by relying on the QR-decomposition, an efficient and robust method is produced to compute explicitly the right Moore-Penrose generalized inverse of the coefficient matrix. The methodology is illustrated via a case study involving a redundantly-actuated parallel-kinematics machine with three degrees of freedom and four actuators.
\end{abstract}

Keywords: Parallel-kinematics machine, actuation redundancy, dynamics modeling, natural orthogonal complement

${ }^{*}$ Corresponding author. 


\section{Introduction}

In modeling the dynamics of multibody systems, explicit equations of motion for the system of interest are to be generated using a suitable methodology. Two methods are mostly employed to construct the dynamics equations, namely, Newton-Euler (NE) and Euler-Lagrange (EL) [1-3]. There are also other widely used methods, such as the virtual work principle [4-6], d'Alembert's principle [7], the principle of Hamilton [8], and Kane's formulation [1], among others [9-13], to cope with the aforementioned task. We adopt in the paper the NE equations to formulate the system of $p$ coupled equations of motion free of constraint wrenches, where $p$ represents the degree of freedom (dof) of the mechanical system at hand. The wrenches at stake are eliminated by means of an orthogonal complement of the matrix of constraints linear in the twist of the individual links. Orthogonal complements have been applied in the realm of mechanical systems since the seventies [14-17]. In the early application of the concept, orthogonal complements were determined by purely algebraic means, not considering the physics involved, which led to orthogonal complements with entries having additions of quantities with different units, as first pointed out by Lipkin [18]. To cope with this issue, the Natural Orthogonal Complement (NOC) [2,19], based on the reciprocity relations between feasible twists and constraint wrenches, was introduced. The NOC, owing its name to the forgoing natural reciprocity relations, allows the elimination of the constraint wrenches upon multiplication of the unconstrained dynamics equations by an orthogonal complement of the velocity constraints. Therefore, compared to the classic NE method, the advantages of this method lie in its systematic computational procedure and low number of intervening variables.

It has been shown that, compared to their non-redundant counterparts, redundantly actuated mechanisms help cope with singularities, besides improving the stiffness of their structures [7,20,21]. As a result, there are many applications of actuation redundancy in a variety of application fields, such as haptic systems [22], parallel kinematics machines [23,24], and medical devices [25]. The NOC methodology can still be applied systematically with inclusion of actuation redundancy. The dynamics modeling of redundantly actuated mechanisms, however, poses an inherent challenge as a result of their closedloop structures. Redundant actuation implies that the actuator torques required, at any instant, to drive the robot throughout a prescribed trajectory, are not unique. This is reflected in theunderdeterminacy of the inverse-dynamics equations in the actuator torques. Underdeterminacy means that the torque requirement at every instant is not unique. The solution is generally solved by resorting to optimization strategies in which an objective function is defined and minimized. The obvious strategy is to choose the actuator-torque array, $\tau$, of minimum cost, i.e., of minimum norm. Of the various norms available, we choose the Euclidean norm, because it lends itself to a closed-form solution, as given by the right Moore-Penrose generalized inverse [26]. This solution is henceforth referred to as the minimum-norm solution.

The paper is organized as follows: We recall the dynamics equations of mechanical systems at large in Section 2, followed by the extension of the modeling to redundantly actuated systems in Section 3. The outcome is an underdetermined system of linear algebraic equations in the actuator torques. The optimum actuator-torque distribution is solved numerically, via the right Moore-Penrose generalized inverse, by means of the QR-decomposition applied to the transpose of the matrix coefficient of the system. In Section 4, the proposed methodology is illustrated in a case study involving a $2 \underline{P} U R-2 R U^{1}$ parallel robot. The paper ends with the Conclusions included in Section 5.

This paper is an extended version of a paper [27], to be presented at the 9th ECCOMAS Thematic Conference on Multibody Dynamics 2019, 15th-18th July 2019, Duisburg, Germany.

\section{Model Formulation}

\subsection{Preliminary Definitions}

It is assumed that the mechanical system at hand is composed of $n$ rigid bodies. The system has $r$ redundantly-actuated joints, its degree of freedom being $p<r$ and its degree of redundancy (dor) $r-p$. Referring to the motion of the $i$ th body, a few concepts are introduced. The six-dimensional twist $\mathbf{t}_{i}$ of and the wrench $\mathbf{w}_{i}$ acting on the $i$ th body are:

$$
\mathbf{t}_{i} \equiv\left[\omega_{i}^{T} \dot{\mathbf{c}}_{i}^{T}\right]^{T} \quad \text { and } \quad \mathbf{w}_{i} \equiv\left[\mathbf{n}_{i}^{T} \mathbf{f}_{i}^{T}\right]^{T}, \quad i=1, \ldots, n
$$

where $\omega_{i}$ and $\dot{\mathbf{c}}_{i}$ are the angular velocity of the body and the velocity of its center of mass, respectively, while $\mathbf{n}_{i}$ and $\mathbf{f}_{i}$ are the moment about and the force applied at the center of mass of the body, correspondingly. Next, the wrench acting on the $i$ th body is decomposed into a generalized external working wrench $\mathbf{w}_{i}^{E}$ and a generalized nonworking constraint wrench $\mathbf{w}_{i}^{C}$, i.e.,

$$
\mathbf{w}_{i}=\mathbf{w}_{i}^{E}+\mathbf{w}_{i}^{C}
$$

\footnotetext{
${ }^{1} \mathrm{P}$ stands for prismatic, $\mathrm{R}$ for revolute, and $\mathrm{U}$ for universal joint, actuated joints being underlined.
} 
Then, the $6 \times 6$ angular velocity dyad $\mathbf{W}_{i}$ and inertia dyad ${ }^{2} \mathbf{M}_{i}$ of the $i$ th body are defined as

$$
\mathbf{W}_{i} \equiv\left[\begin{array}{cc}
\Omega_{i} & \mathbf{O} \\
\mathbf{O} & \mathbf{O}
\end{array}\right] \quad \text { and } \quad \mathbf{M}_{i} \equiv\left[\begin{array}{cc}
\mathbf{I}_{C i} & \mathbf{O} \\
\mathbf{O} & m_{i} \mathbf{1}
\end{array}\right]
$$

where $\Omega_{i}$ is the $3 \times 3$ cross-product matrix $(\mathrm{CPM})^{3}$ of vector $\omega_{i}$. Moreover, $\mathbf{O}$ and $\mathbf{1}$ are the $3 \times 3$ zero and identity matrices, respectively, while $\mathbf{I}_{C i}$ is the $3 \times 3$ inertia tensor about the center of mass of the $i$ th body, and $m_{i}$ the mass of the body.

N.B.: Henceforth, we refer to the above concepts, twist and wrench, along with its germane concepts, angular velocity dyad and its inertia counterpart, as Cartesian variables.

\subsection{Single-body Dynamics Equations}

The Newton-Euler equations of motion of the $i$ th body are recalled below:

$$
\begin{array}{r}
\mathbf{I}_{C i} \omega_{i}+\omega_{i} \times \mathbf{I}_{C i} \omega_{i}=\mathbf{n}_{i} \\
m_{i} \ddot{\mathbf{c}}_{i}=\mathbf{f}_{i}
\end{array}
$$

With the definitions introduced in Section 2.1, the foregoing equations are cast in a compact form as [2]:

$$
\mathbf{M}_{i} \dot{\mathbf{t}}_{i}=-\mathbf{W}_{i} \mathbf{M}_{i} \mathbf{t}_{i}+\mathbf{w}_{i}^{E}+\mathbf{w}_{i}^{C}, \quad i=1, \ldots, n
$$

Let $\mathbf{x} \equiv\left[x_{1}, \ldots, x_{p}\right]^{T}$ represent the generalized coordinates of the system, its time-rate of change $\dot{\mathbf{x}} \equiv\left[\dot{x}_{1}, \ldots, \dot{x}_{p}\right]^{T}$ denoting the array of generalized velocities of the same. The twist of the $i$ th body is obtained as

$$
\mathbf{t}_{i}=\mathbf{T}_{i} \dot{\mathbf{x}}, \quad i=1, \ldots, n
$$

where $\mathbf{T}_{i}$ is the $6 \times p$ twist-shaping matrix of the $i$ th body, which maps $\dot{\mathbf{x}}$ into the twist array of the $i$ th body. Note that the power developed by the constraint wrench $\mathbf{w}_{i}^{C}$ on the feasible twist $\mathbf{t}_{i}$ vanishes, i.e.,

$$
\mathbf{t}_{i}^{T} \mathbf{w}_{i}^{C}=0
$$

Upon substitution of Eq. (6) into Eq. (7), we obtain

$$
\dot{\mathbf{x}}^{T} \mathbf{T}_{i}^{T} \mathbf{w}_{i}^{C}=0
$$

Since Eq. (8) must be satisfied for arbitrary values of the generalized velocities $\dot{\mathbf{x}}$, because they are independent, the product $\mathbf{T}_{i}^{T} \mathbf{w}_{i}^{C}$ must vanish. Therefore, upon multiplying both sides of Eq. (5) by $\mathbf{T}_{i}^{T}$, we obtain

$$
\mathbf{T}_{i}^{T} \mathbf{M}_{i} \dot{\mathbf{t}}_{i}=-\mathbf{T}_{i}^{T} \mathbf{W}_{i} \mathbf{M}_{i} \dot{\mathbf{t}}_{i}+\mathbf{T}_{i}^{T} \mathbf{w}_{i}^{E}, \quad i=1, \ldots, n
$$

Notice that, for the $i$ th body, the external wrench $\mathbf{w}_{i}^{E}$ arises from all forces and moments exerted on the body by its environment, namely, actuator wrenches $\mathbf{w}_{i}^{A}$, gravity wrenches $\mathbf{w}_{i}^{G}$, and dissipation wrenches $\mathbf{w}_{i}^{D}$ :

$$
\mathbf{w}_{i}^{E}=\mathbf{w}_{i}^{A}+\mathbf{w}_{i}^{G}+\mathbf{w}_{i}^{D}
$$

Hence, upon differentiation of both sides of Eq. (6) with respect to time,

$$
\dot{\mathbf{t}}_{i}=\mathbf{T}_{i} \ddot{\mathbf{x}}+\dot{\mathbf{T}}_{i} \dot{\mathbf{x}}, \quad i=1, \ldots, n
$$

\footnotetext{
${ }^{2}$ Here we adopt the term, "dyad", first introduced by von Mises [28], to represent the inertia properties of a rigid body, mass and moment of inertia, in one single $6 \times 6$ array, displayed as $\mathbf{M}_{i}$ in Eq. (3).

${ }^{3}$ The CPM $\mathbf{V} \in \mathbb{R}^{3 \times 3}$ of any vector $\mathbf{v} \in \mathbb{R}^{3}$, is defined as $\mathbf{V}=(\partial \mathbf{v} \times \mathbf{x} / \partial \mathbf{x}), \forall \mathbf{x} \in \mathbb{R}^{3}$.
} 
Finally, when Eqs. (10) and (11) are substituted into Eq. (9), the Newton-Euler equations of the $i$ th body are obtained in the domain of the generalized coordinates, as opposed to those in Eq. (9), expressed in the domain of Cartesian variables, twist and wrench associated with the same body, namely,

$$
\mathbf{I}_{i} \ddot{\mathbf{x}}+\mathbf{C}_{i} \dot{\mathbf{x}}=\mathbf{T}_{i}^{T} \mathbf{w}_{i}^{A}+\mathbf{T}_{i}^{T} \mathbf{w}_{i}^{G}+\mathbf{T}_{i}^{T} \mathbf{w}_{i}^{D}, \quad i=1, \ldots, n
$$

where $\mathbf{I}_{i}$ denotes the $6 \times 6$ inertia dyad of the $i$ th body and $\mathbf{C}_{i}$ the $6 \times 6$ dyad of Coriolis and centrifugal terms of the same body, namely,

$$
\mathbf{I}_{i} \equiv \mathbf{T}_{i}^{T} \mathbf{M}_{i} \mathbf{T}_{i}, \quad \mathbf{C}_{i} \equiv \mathbf{T}_{i}^{T}\left(\mathbf{M}_{i} \dot{\mathbf{T}}_{i}+\mathbf{W}_{i} \mathbf{M}_{i} \mathbf{T}_{i}\right), \quad i=1, \ldots, n
$$

\subsection{Mutlibody Dynamics Equations}

We formulate here the mathematical model of the whole system free of constraint wrenches. The latter are eliminated by virtue of the reciprocity relations between system twist and system wrench, concepts that are defined in the same subsection.

Global concepts, pertaining to the whole system, are now introduced: system twist $\mathbf{t}$; system constraint wrench $\mathbf{w}^{C}$; system actuator wrench $\mathbf{w}^{A}$; system gravity wrench $\mathbf{w}^{G}$; and system dissipation wrench, $\mathbf{w}^{D}$. Additionally, the $6 n \times 6 n$ blockdiagonal matrices of system mass $\mathbf{M}$ and system angular velocity $\mathbf{W}$, along with the $6 n \times p$ system twist-shaping matrix $\mathbf{T}$, are also introduced:

$$
\begin{aligned}
\mathbf{t} & =\left[\begin{array}{c}
\mathbf{t}_{1} \\
\vdots \\
\mathbf{t}_{n}
\end{array}\right], \quad \mathbf{T}=\left[\begin{array}{c}
\mathbf{T}_{1} \\
\vdots \\
\mathbf{T}_{n}
\end{array}\right], \quad \mathbf{w}^{C}=\left[\begin{array}{c}
\mathbf{w}_{1}^{C} \\
\vdots \\
\mathbf{w}_{n}^{C}
\end{array}\right] \\
\mathbf{w}^{A} & =\left[\begin{array}{c}
\mathbf{w}_{1}^{A} \\
\vdots \\
\mathbf{w}_{n}^{A}
\end{array}\right], \quad \mathbf{w}^{G}=\left[\begin{array}{c}
\mathbf{w}_{1}^{G} \\
\vdots \\
\mathbf{w}_{n}^{G}
\end{array}\right], \quad \mathbf{w}^{D}=\left[\begin{array}{c}
\mathbf{w}_{1}^{D} \\
\vdots \\
\mathbf{w}_{n}^{D}
\end{array}\right] \\
\mathbf{M} & =\operatorname{diag}\left(\mathbf{M}_{1}, \ldots, \mathbf{M}_{n}\right) \\
\mathbf{W} & =\operatorname{diag}\left(\mathbf{W}_{1}, \ldots, \mathbf{W}_{n}\right)
\end{aligned}
$$

Then, the unconstrained system dynamics equations take the form as:

$$
\mathbf{M} \dot{\mathbf{t}}=-\mathbf{W M t}+\mathbf{w}^{A}+\mathbf{w}^{G}+\mathbf{w}^{D}+\mathbf{w}^{C}
$$

The kinematic constraints of the system can be formulated as a linear homogeneous system in the $6 n$-dimensional system twist $\mathbf{t}$, as shown elsewhere [19]:

$$
\mathbf{K t}=\mathbf{0}
$$

where $\mathbf{K}$ is a $6 n \times 6 n$ matrix of rank $6 n-p$, and the system twist is obtained as:

$$
\mathbf{t}=\mathbf{T} \dot{\mathbf{x}}
$$

Upon substitution of Eq. (17) into Eq. (16), we can readily derive:

$$
\mathbf{K T}=\mathbf{O}
$$

where $\mathbf{O}$ is the $6 n \times p$ zero matrix, which shows that $\mathbf{T}$ is an orthogonal complement of $\mathbf{K}$. In fact, upon transposing the two sides of the foregoing equation, an equivalent relation is derived, showing that, correspondingly, $\mathbf{K}^{T}$ is an orthogonal complement of $\mathbf{T}^{T}$. By virtue of the foregoing analysis, that led to Eq. (18), upon resorting to the reciprocity relations between feasible twists and constraint wrenches in a mechanical system of rigid bodies, $\mathbf{K}$ is termed the natural orthogonal complement of $\mathbf{T}$. 
Furthermore, upon differentiation of both sides of Eq. (17) with respect to time, an expression for the time-rate of change of the system twist $\mathbf{t}$ is obtained:

$$
\dot{\mathbf{t}}=\mathbf{T} \ddot{\mathbf{x}}+\dot{\mathbf{T}} \dot{\mathbf{x}}
$$

Then, upon substitution of above equation into Eq. (15), followed by premultiplication of the two sides of the equation thus resulting $\mathbf{T}^{T}$, while considering that

$$
\mathbf{T}^{T} \mathbf{w}^{C} \equiv \sum_{i=1}^{n} \mathbf{T}_{i}^{T} \mathbf{w}_{i}^{C}=0
$$

the Newton-Euler equations of the system are obtained in the domain of the generalized coordinates, as opposed to those in Eq. (9), expressed in the domain of Cartesian variables, twist and wrench associated with the system bodies, namely,

$$
\mathbf{I} \ddot{\mathbf{x}}+\mathbf{C} \dot{\mathbf{x}}=\mathbf{T}^{T} \mathbf{w}^{A}+\mathbf{T}^{T} \mathbf{w}^{G}+\mathbf{T}^{T} \mathbf{w}^{D}
$$

where

$$
\begin{aligned}
\mathbf{I} & \equiv \mathbf{T}^{T} \mathbf{M} \mathbf{T} \equiv \sum_{i=1}^{n} \mathbf{T}_{i}^{T} \mathbf{M}_{i} \mathbf{T}_{i} \\
\mathbf{C} & \equiv \mathbf{T}^{T}(\mathbf{M} \mathbf{T}+\mathbf{W M T}) \equiv \sum_{i=1}^{n} \mathbf{T}_{i}^{T}\left(\mathbf{M}_{i} \dot{\mathbf{T}}_{i}+\mathbf{W}_{i} \mathbf{M}_{i} \mathbf{T}_{i}\right)
\end{aligned}
$$

Now, let $\tau, \gamma$, and $\delta$ denote the $p$-dimensional vectors of actuator, gravity and dissipation generalized forces exerted on the system, i.e.,

$$
\begin{gathered}
\tau \equiv \mathbf{T}^{T} \mathbf{w}^{A} \equiv \sum_{i=1}^{n} \mathbf{T}_{i}^{T} \mathbf{w}_{i}^{A} \\
\gamma \equiv \mathbf{T}^{T} \mathbf{w}^{G} \equiv \sum_{i=1}^{n} \mathbf{T}_{i}^{T} \mathbf{w}_{i}^{G} \\
\delta \equiv \mathbf{T}^{T} \mathbf{w}^{D} \equiv \sum_{i=1}^{n} \mathbf{T}_{i}^{T} \mathbf{w}_{i}^{D}
\end{gathered}
$$

Thereby obtaining the mathematical model sought:

$$
\tau=\mathbf{I} \ddot{\mathbf{x}}+\mathbf{C} \dot{\mathbf{x}}-\gamma-\delta
$$

Considering that the angular velocity dyad $\mathbf{W}_{i}$ is skew-symmetric and the inertia dyad $\mathbf{M}_{i}$ is symmetric, it can be readily shown that I is symmetric. Moreover,

$$
\begin{aligned}
\dot{\mathbf{I}} & =\sum_{i=1}^{n} \dot{\mathbf{T}}_{i}^{T} \mathbf{M}_{i} \mathbf{T}_{i}+\sum_{i=1}^{n} \mathbf{T}_{i}^{T} \dot{\mathbf{M}}_{i} \mathbf{T}_{i}+\sum_{i=1}^{n} \mathbf{T}_{i}^{T} \mathbf{M}_{i} \dot{\mathbf{T}}_{i} \\
& =\sum_{i=1}^{n} \dot{\mathbf{T}}_{i}^{T} \mathbf{M}_{i} \mathbf{T}_{i}+\sum_{i=1}^{n} \mathbf{T}_{i}^{T}\left(\mathbf{W}_{i} \mathbf{M}_{i}-\mathbf{M}_{i} \mathbf{W}_{i}\right) \mathbf{T}_{i}+\sum_{i=1}^{n} \mathbf{T}_{i}^{T} \mathbf{M}_{i} \dot{\mathbf{T}}_{i}
\end{aligned}
$$

Thus,

$$
\dot{\mathbf{I}}-2 \mathbf{C}=\underbrace{\sum_{i=1}^{n} \dot{\mathbf{T}}_{i}^{T} \mathbf{M}_{i} \mathbf{T}_{i}-\sum_{i=1}^{n} \mathbf{T}_{i}^{T} \mathbf{M}_{i} \dot{\mathbf{T}}_{i}}_{\text {skew-symmetric }}-\underbrace{\sum_{i=1}^{n} \mathbf{T}_{i}^{T}\left(\mathbf{W}_{i} \mathbf{M}_{i}+\mathbf{M}_{i} \mathbf{W}_{i}\right) \mathbf{T}_{i}}_{\text {skew-symmetric }}
$$


thereby verifying two basic results in the realm of mechanical systems of rigid bodies:

1. I is symmetric and positive definite.

2. $\mathbf{I}-2 \mathbf{C}$ is skew-symmetric.

This result leads to the controllability of a multibody system, holonomic or nonholonomic, with a linear PD controller. Indeed, any control law methodologies available for serial robots can be applied to their parallel counterparts because of the skew-symmetry of $\mathbf{I}-2 \mathbf{C}$ [29]. It is noteworthy that care should be taken upon application of control schemes to redundantly-actuated systems, as significantly large internal forces can be generated [30]. Moreover, play and wear are always present [31], but not considered in the model, their existence preventing application of pure position-control schemes. Therefore, typically, a force/position scheme is recommended to control multibody mechanical systems.

\section{The Computation of the Optimum Actuator-torque Distribution}

Let $\mathbf{q} \equiv\left[q_{1}, \ldots, q_{r}\right]^{T}$ be the array of the actuated-joint coordinates, and $\dot{\mathbf{q}} \equiv\left[\dot{q}_{1}, \ldots, \dot{q}_{r}\right]^{T}$ the array of the actuated-joint rates. By virtue of the Principle of Conservation of Energy, the power delivered by the actuators at every instant equals its counterpart, developed by the robot links, in generalized space, i.e.,

$$
\tau_{a}^{T} \dot{\mathbf{q}}=\tau^{T} \dot{\mathbf{x}}
$$

where $\tau_{a}$ is the array of the actuator torques. Moreover, the $r \times p$ matrix $\mathbf{A}$, that maps $\dot{\mathbf{x}}$ into $\dot{\mathbf{q}}$, is introduced as

$$
\dot{\mathbf{q}}=\mathbf{A} \dot{\mathbf{x}}
$$

Upon substitution of Eq. (28) into Eq. (27), we obtain

$$
\tau_{a}^{T} \mathbf{A} \dot{\mathbf{x}}=\tau^{T} \dot{\mathbf{x}}
$$

Now, the generalized rates stored in array $\dot{\mathbf{x}}$ are independent, and hence, can be assigned arbitrarily. Therefore, $\dot{\mathbf{x}}$ can be "deleted" from Eq. (29), which leads to

$$
\tau=\mathbf{A}^{T} \tau_{a}
$$

which is a system of $p$ equations in $\tau_{a}$ for $r>p$ components of $\tau_{a}$. Hence, given any value of $\tau$ in Eq. (30), to carry the robot through a given Cartesian trajectory, infinitely-many values of $\tau_{a}$ are available for the task at hand, a result of redundant actuation. Under the foregoing conditions, the obvious strategy is to choose the actuator-torque (or force) array of minimum cost, which means of minimum norm ${ }^{4}$, whatever the norm of choice is. Of the various norms available, we opt for the Euclidean norm, which allows for a closed-form expression of the actuator-torque (or force) array $\tau_{a}$ in terms of the right Moore-Penrose generalized inverse of $\mathbf{A}^{T}$. In the paper we assume that matrix $\mathbf{A}$ is of full rank. This is the case if no two actuators are duplicated, as in the case of two rotational actuators mounted on the same axis. Symbolically, the minimum-norm solution sought is expressed as [32]:

$$
\tau_{a 0}=\mathbf{A}^{\dagger} \tau
$$

where

$$
\mathbf{A}^{\dagger}=\mathbf{A}\left(\mathbf{A}^{T} \mathbf{A}\right)^{-1}
$$

which is the right Moore-Penrose generalized inverse (RMPGI) of the rectangular matrix $\mathbf{A}^{T}$. It can be seen that the straightforward evaluation of $\mathbf{A}^{\dagger}$ involves the inversion of the product $\mathbf{A}^{T} \mathbf{A}$, which is not only computationally costly, but also prone to ill-conditioning ${ }^{5}$ [26].

\footnotetext{
${ }^{4}$ In the case of a combination of rotational and translational actuators, the norm in question must be suitably weighted, so as to allow for a physically meaningful norm.

${ }^{5}$ The condition number of the product is, roughly, the square of the condition number of $\mathbf{A}$.
} 
The way to compute robustly the minimum-norm solution $\tau_{a 0}$ is by means of the QR-decomposition of matrix $\mathbf{A}$ [26], namely,

$$
\mathbf{A}=\mathbf{Q R}
$$

where $\mathbf{Q}$ is $r \times r$ orthogonal matrix and $\mathbf{R}$ is a $r \times p$ upper-triangular matrix, with $r>p$. Moreover, the above factoring is obtained by means of $r$ Householder reflections [26], i.e., improper orthogonal matrices ${ }^{6}$. That is, if $\mathbf{Q}$ and $\mathbf{R}$ are expressed in block form:

$$
\mathbf{Q}=\left[\begin{array}{ll}
\mathbf{Q}_{L} & \mathbf{Q}_{R}
\end{array}\right], \quad \mathbf{R}=\left[\begin{array}{c}
\mathbf{U} \\
\mathbf{O}_{r^{\prime} p}
\end{array}\right], \quad r^{\prime}=r-p
$$

then $\mathbf{A}^{\dagger}$ becomes

$$
\mathbf{A}^{\dagger}=\mathbf{Q}_{L} \mathbf{U}^{-T}
$$

and hence, the minimum-norm solution $\tau_{a 0}$ becomes

$$
\tau_{a 0}=\mathbf{Q}_{L} \mathbf{U}^{-T}(\mathbf{I} \ddot{\mathbf{x}}+\mathbf{C} \dot{\mathbf{x}}-\boldsymbol{\gamma}-\boldsymbol{\delta})
$$

The basic concepts and steps of the proposed modeling method are shown in Fig. 1. The procedure will be illustrated with an application to a redundantly actuated parallel robot with three dof and four actuators. Since the aforementioned modeling approach is generally applicable to redundantly actuated systems with dor $=r-p$, it should be applicable to hyper-redundant systems as well.

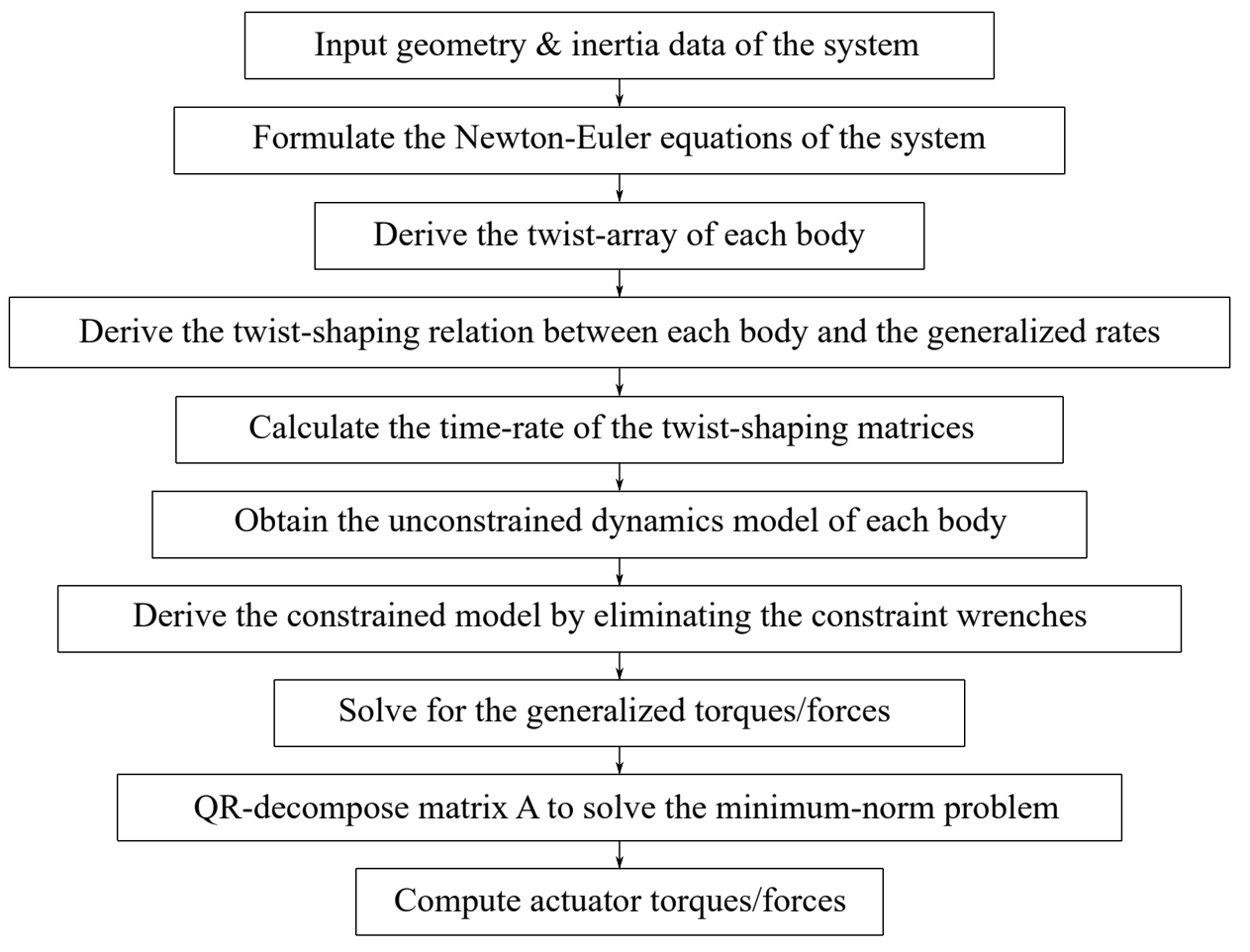

Fig. 1: Basic concepts and modeling logic

\footnotetext{
${ }^{6}$ These are defined as orthogonal matrices whose determinant is -1 , as opposed to their proper counterparts, of determinant +1 , which represent rotations.
} 
Table 1: Robot Geometric Data

\begin{tabular}{l|ccc}
\hline Description & Symbol & Value & Units \\
\hline Limb $B_{i} A_{i}$ length $(i=1,2)$ & $l$ & 120 & $\mathrm{~mm}$ \\
$O C_{i}$ length $(i=3,4)$ & $l_{3}$ & 80 & $\mathrm{~mm}$ \\
$P A_{i}$ length $(i=1, \ldots, 4)$ & $f$ & 60 & $\mathrm{~mm}$ \\
$C_{i} B_{i}$ length $(i=3,4)$ & $d$ & 20 & $\mathrm{~mm}$ \\
Distance between $A_{1} A_{2}$ and $A_{3} A_{4}$ & $e$ & 0 & $\mathrm{~mm}$ \\
\hline
\end{tabular}

\section{Case Study: The 2PUR-2RPU Parallel Robot}

\subsection{Robot Description}

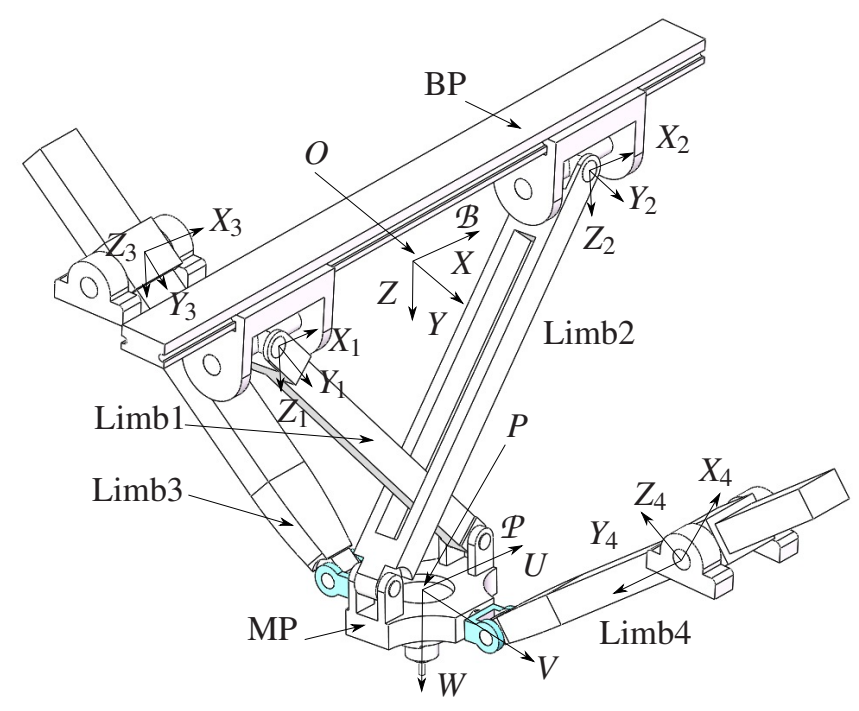

Fig. 2: 2UR-2R $\underline{P U}$ robot

The foregoing methodology is now illustrated and applied to a redundantly-actuated PKM. The machine under study is of the 2PUR-2RPU 2R1T ${ }^{7}$ type [21], as shown in Figs. 2 and 3, its geometric parameters being listed in Table 1. The robot is composed of a base platform (BP), two identical PUR and two identical RPU limbs, each pair actuated at its respective $\mathrm{P}$ joint, and a moving platform (MP). The $i$ th limb for $i=1,2,3,4$, is $A_{i} B_{i} C_{i}$. A Cartesian coordinate frame, $\mathcal{B}(X, Y, Z)$, is attached to the fixed base at the intersection $O$ of lines $B_{1} B_{2}$ and $C_{3} C_{4}$. A moving coordinate frame, $\mathcal{P}(U, V, W)$, is attached to the moving platform at $P$, the midpoint of the line segment $\overline{A_{3} A_{4}}$. The platform is capable of two rotations, through angles $\alpha$ and $\beta$, about corresponding skew axes, $X$ and $V$, at right angles, and one translation, $\zeta$, along vector $\mathbf{p}=\overrightarrow{O P}$, normal to the $V$-axis.

In the machining of workpieces with complex curved surfaces, such lower-mobility 2R1T PKMs, integrated either with a two-to-three-dof tool head or a two-dof gantry to form a five-to-six-dof hybrid PKM, have been considerably researched and subsequently applied [33-37]. These applications require high speed and high precision, hence the importance of the dynamics modeling and analysis.

In the definitions below, for $i=1, \ldots, 7$, the $i$ th link of the system denotes, in the given order: the moving link of the $\mathrm{P}$ joint of the first limb; link $B_{1} A_{1}$; the moving link of the $\mathrm{P}$ joint of the second limb; link $B_{2} A_{2}$; link $B_{3} A_{3}$; link $B_{4} A_{4}$; and the MP. Furthermore, for $i=1,2, l_{c}$ denotes the distance from the center of mass of link $B_{i} A_{i}$ to the center of the corresponding $\mathrm{U}$ joint, $B_{i}$, while $q_{c}$ denotes the distance from the center of mass of link $B_{i} A_{i}$, for $i=3,4$, to the center of the corresponding U-joint, $A_{i}$. Furthermore, let $\theta_{i}$ denote the angle between $Z_{i}$ and $\overrightarrow{B_{i} A_{i}}$ for $i=1,2$, and between $Y_{i}$ and $Y$ for $i=3,4$. A tool,

\footnotetext{
${ }^{7} \mathrm{~A}$ PKM with two rotational and one translational dof.
} 


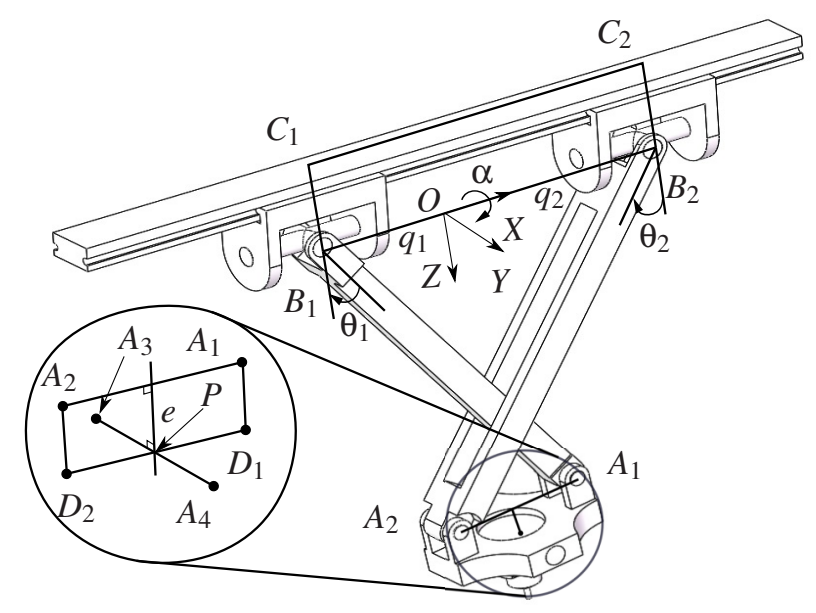

(a) PURRUP chain of limbs $1 \& 2$

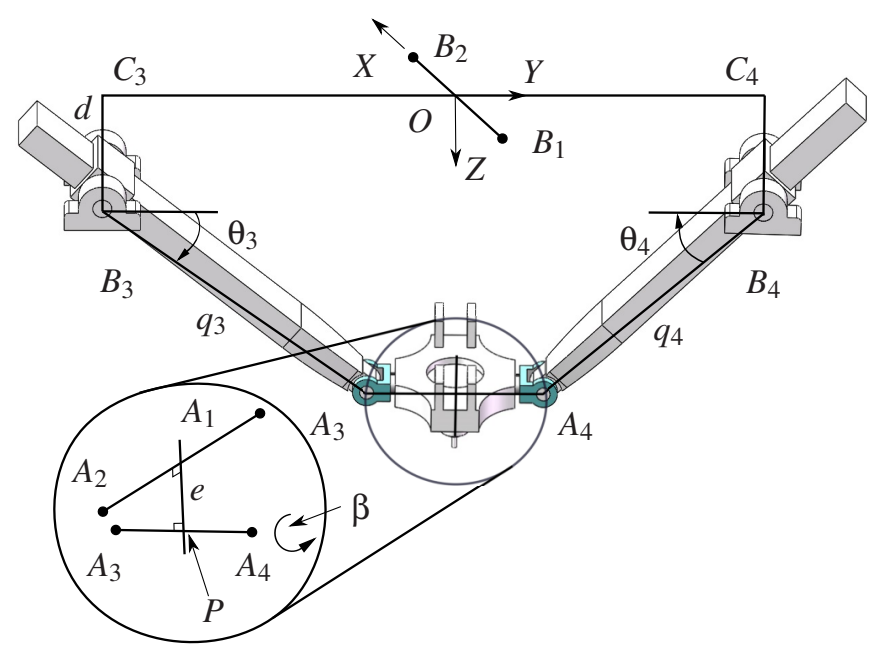

(b) RPUUPR chain of limbs 3 \& 4

Fig. 3: Dimensions of the 2PUR-2R $\underline{P} U$ robot

of length $h$, is added at the reference point $P$ in the MP. The tool length is measured from $P$ along the axis $W$, in its positive direction.

\subsection{Twist Arrays of the Robot Links}

Coupled by a revolute or prismatic joint, the relative gesture of two rigid links, i.e., the twist of link 2 with respect to link 1, can be expressed as a corresponding screw of the revolute or the prismatic pair involved. The twists, $\mathbf{t}_{r}$ and $\mathbf{t}_{p}$, of the relative gesture of two rigid links coupled by the the revolute and prismatic pairs, respectively, are given by

$$
\mathbf{t}_{r}=\dot{\theta}\left[\begin{array}{c}
\mathbf{e}_{1} \\
\mathbf{e}_{1} \times \mathbf{s}
\end{array}\right], \quad \mathbf{t}_{p}=\dot{b}\left[\begin{array}{c}
\mathbf{0} \\
\mathbf{e}_{2}
\end{array}\right]
$$

where, for the revolute joint, $\mathbf{e}_{1}$ is the axis of rotation, $\theta$ the angle of rotation, and $\mathbf{s}$ the vector directed from a point $O$ of the axis to the center of mass of link 2; for the prismatic joint, $b$ is the translation, and $\mathbf{e}_{2}$ the direction of translation. Thus, the twist $\mathbf{t}$ of the end link of a serial chain of $j$ links can be obtained as a combination of consecutive twists:

$$
\mathbf{t}=\sum_{i=1}^{j} \mathbf{t}_{i}
$$


where $\mathbf{t}_{i}$ denotes the relative twist of link $i$ with respect to its parent link $i-1$.

Given the generalized coordinates array of $\mathbf{x} \equiv[\alpha, \beta, \zeta]^{T}$, the inverse-displacement solution, i.e., the array of the actuated-joint coordinates $\mathbf{q} \equiv\left[q_{1}, q_{2}, q_{3}, q_{4}\right]^{T}$, is recalled:

$$
\mathbf{q}=\left[\begin{array}{l}
q_{1} \\
q_{2} \\
q_{3} \\
q_{4}
\end{array}\right]=\left[\begin{array}{c}
-g_{11}+f_{1} \cos \beta-e \sin \beta \\
g_{21}-f_{1} \cos \beta-e \sin \beta \\
\sqrt{g_{31}^{2}+g_{32}^{2}} \\
\sqrt{g_{41}^{2}+g_{42}^{2}}
\end{array}\right]
$$

Thus, using the aforementioned procedure, the six-dimensional twists of the system bodies are obtained as:

$$
\mathbf{t}_{1}=\left[0,0,0, \dot{q}_{1}, 0,0\right]^{T}, \quad \mathbf{t}_{2}=\left[0,0,0, \dot{q}_{2}, 0,0\right]^{T}
$$

$$
\mathbf{t}_{3}=\left[\begin{array}{c}
\dot{\alpha} \\
\cos \alpha \dot{\theta}_{1} \\
\sin \alpha \dot{\theta}_{1} \\
l_{c} \cos \theta_{1} \dot{\theta}_{1}+\dot{q}_{1} \\
l_{c}\left(-\cos \alpha \cos \theta_{1} \dot{\alpha}+\sin \alpha \sin \theta_{1} \dot{\theta}_{1}\right) \\
-l_{c}\left(\sin \alpha \cos \theta_{1} \dot{\alpha}+\cos \alpha \sin \theta_{1} \dot{\theta}_{1}\right)
\end{array}\right]
$$

$$
\mathbf{t}_{4}=\left[\begin{array}{c}
\dot{\alpha} \\
\cos \alpha \dot{\theta}_{2} \\
\sin \alpha \dot{\theta}_{2} \\
l_{c} \cos \theta_{2} \dot{\theta}_{2}+\dot{q}_{2} \\
l_{c}\left(-\cos \alpha \cos \theta_{2} \dot{\alpha}+\sin \alpha \sin \theta_{2} \dot{\theta}_{2}\right) \\
-l_{c}\left(\sin \alpha \cos \theta_{2} \dot{\alpha}+\cos \alpha \sin \theta_{2} \dot{\theta}_{2}\right)
\end{array}\right]
$$

$$
\mathbf{t}_{5}=\left[\begin{array}{c}
\dot{\theta}_{3} \\
0 \\
0 \\
0 \\
\left(q_{c}-q_{3}\right) \sin \theta_{3} \dot{\theta}_{3}+\cos \theta_{3} \dot{q}_{3} \\
\left(q_{3}-q_{c}\right) \cos \theta_{3} \dot{\theta}_{3}+\sin \theta_{3} \dot{q}_{3}
\end{array}\right]
$$

$$
\mathbf{t}_{6}=\left[\begin{array}{c}
\dot{\theta}_{4} \\
0 \\
0 \\
0 \\
\left(q_{c}-q_{4}\right) \sin \theta_{4} \dot{\theta}_{4}+\cos \theta_{4} \dot{q}_{4} \\
\left(q_{4}-q_{c}\right) \cos \theta_{4} \dot{\theta}_{4}+\sin \theta_{4} \dot{q}_{4}
\end{array}\right]
$$

$$
\mathbf{t}_{7}=[\dot{\alpha}, \cos \alpha \dot{\beta}, \sin \alpha \dot{\beta}, \sin \beta \dot{\zeta},-\sin \alpha \cos \beta \dot{\zeta}, \cos \alpha \cos \beta \dot{\zeta}]^{T}
$$


Table 2: Computational cost of the dynamics model

\begin{tabular}{l|cc}
\hline Description & Symbol & Flops \\
\hline Number of flops to compute $\theta_{i}$ & $N_{\theta_{i}}$ & $19 \times 4$ \\
Number of flops to compute $g_{i j}$ & $N_{g_{i j}}$ & 33 \\
Number of flops to compute $k_{i j}$ & $N_{k_{i j}}$ & 74 \\
Number of flops to compute $\mathbf{T}_{i}$ & $N_{T_{i}}$ & 90 \\
Number of flops to compute $\mathbf{T}_{i}$ & $N_{\mathbf{T}_{i}}$ & $7 \times 18$ \\
Number of flops to compute $I$ & $N_{I}$ & $7 \times 72$ \\
Number of flops to compute $C$ & $N_{C}$ & $7 \times 145$ \\
Number of flops to compute $\gamma$ & $N_{\gamma}$ & $7 \times 18$ \\
Number of flops to compute $\tau$ & $N_{\tau}$ & 18 \\
Number of flops to compute $\tau_{a 0}$ & $N_{\tau_{a 0}}$ & 63 \\
Total computational cost & $N_{I D A}$ & 2125 \\
\hline
\end{tabular}

\subsection{Twist-shaping Relations}

The twist-shaping matrix, mapping the three-dimensional array of generalized rates into the link twist, is derived by calculation of the partial derivative of each twist with respect to the generalized rates array $\dot{\mathbf{x}} \equiv[\dot{\alpha}, \dot{\beta}, \dot{\zeta}]^{T}$. Therefore, in this case, the twist-shaping matrices are all $6 \times 3$ arrays, obtained as

$$
\mathbf{T}_{i} \equiv \frac{\partial \mathbf{t}_{i}}{\partial \dot{\mathbf{x}}}, \quad i=1, \ldots, 7
$$

The array expressions for the seven twist-shaping matrices being rather bulky, they are displayed in the Appendix ${ }^{8}$.

\subsection{Computational Cost}

We recall below the number of flops estimated for the computation of trigonometric functions, harmonic or otherwise $\left(N_{t}\right)$, their inverse $\left(N_{i}\right)$, and square roots $\left(N_{s}\right)$, as reported by Karimi-Eskandary et al. [38]:

$$
N_{t}=18, \quad N_{i}=23, \quad N_{s}=6
$$

The computational cost of the dynamics model can be calculated by counting the total number of flops required to compute the various terms in Eq. (21). Note that items such as inertia dyad $\mathbf{M}_{i}$, the angular-velocity dyad $\mathbf{W}_{i}$, and the wrench vector $\mathbf{w}_{i}$ are either constant or obtained by simple look-up from a data file; hence they do not contribute to the cost. The cost of the other parameters involved is listed in Table 2.

\subsection{Simulation}

An example of the inverse dynamics is included here. Assuming that links and joints are made of 6061-T6 Alloy, with a density of $2700 \mathrm{~kg} / \mathrm{m}^{3}$, the mass of each body and their moment-of-inertia matrices at the respective centers of mass are

\footnotetext{
${ }^{8}$ This is done with the purpose of preserving continuity in the main body of the paper.
} 
listed below:

$$
\begin{aligned}
m_{1} & =3.261 \cdot 10^{-2} \mathrm{~kg} \\
{\left[\mathbf{I}_{1}\right] } & =\operatorname{diag}(4.855,12.742,12.795) \cdot 10^{-6} \mathrm{~kg} \cdot \mathrm{m}^{2} \\
m_{2} & =3.300 \cdot 10^{-2} \mathrm{~kg} \\
{\left[\mathbf{I}_{2}\right] } & =\operatorname{diag}(40.048,39.770,1.054) \cdot 10^{-6} \mathrm{~kg} \cdot \mathrm{m}^{2} \\
m_{3} & =3.261 \cdot 10^{-2} \mathrm{~kg} \\
{\left[\mathbf{I}_{3}\right] } & =\operatorname{diag}(4.855,12.742,12.795) \cdot 10^{-6} \mathrm{~kg} \cdot \mathrm{m}^{2} \\
m_{4} & =4.200 \cdot 10^{-2} \mathrm{~kg} \\
{\left[\mathbf{I}_{4}\right] } & =\operatorname{diag}(52.324,50.786,2.543) \cdot 10^{-6} \mathrm{~kg} \cdot \mathrm{m}^{2} \\
m_{5} & =6.082 \cdot 10^{-2} \mathrm{~kg} \\
{\left[\mathbf{I}_{5}\right] } & =\operatorname{diag}(109.396,3.349,110.827) \cdot 10^{-6} \mathrm{~kg} \cdot \mathrm{m}^{2} \\
m_{6} & =6.082 \cdot 10^{-2} \mathrm{~kg} \\
{\left[\mathbf{I}_{6}\right] } & =\operatorname{diag}(109.396,3.349,110.827) \cdot 10^{-6} \mathrm{~kg} \cdot \mathrm{m}^{2} \\
m_{7} & =8.200 \cdot 10^{-2} \mathrm{~kg} \\
{\left[\mathbf{I}_{7}\right] } & =\operatorname{diag}(16.631,27.155,37.459) \cdot 10^{-6} \mathrm{~kg} \cdot \mathrm{m}^{2}
\end{aligned}
$$

Although line-path or circle-path are two typical types of test trajectories used by the robotics community, a third type, namely, helical-path, which combines line-path and circle-path, is more general and widely used in PKM dynamics analysis [39]. The path to be traced, fixed on the base frame, is given by

$$
\begin{aligned}
& x=r \cos \phi-r \\
& y=r \sin \phi \\
& z=z_{0}+h_{0} \phi /(2 \pi)
\end{aligned}
$$

with the numerical values: $r=5 \mathrm{~mm}$; initial height $z_{0}=127.48 \mathrm{~mm}$; pitch $h_{0}=20 \mathrm{~mm}$; and the angle $0 \leq \phi \leq 2 \pi$.

The trapezoidal velocity trajectory, consisting of three phases: acceleration, coasting, and deceleration, is known for its simplicity, hence very common in the industrial practice. Its acceleration, however, is discontinuous. In order to obtain the continuity of the acceleration profile, we resort to a modified trapezoidal trajectory [40], which is divided into six phases of either second degree polynomial or cycloidal function, as shown in Eq. (B-1), where $T$ is the cycle-time, assumed to be $10 \mathrm{~s}$ in this case.

Furthermore, notice that the mobility of the mechanism under study comprises two rotations about skew axes and one translation along vector $\mathbf{p}=\overrightarrow{O P}$. Under these conditions, the Cartesian coordinates of the operation point of the tool-head, attached to the MP, can still be controlled, if at the sacrifice of the orientation dof, as illustrated below.

Now, the relation between Cartesian coordinates $\mathbf{y} \equiv[x, y, z]^{T}$, of the tool head, and the generalized coordinates $\mathbf{x} \equiv$ $[\alpha, \beta, \zeta]^{T}$, of the MP, is derived as:

$$
\begin{aligned}
& \alpha=\arctan (-y / z) \\
& \beta=\arcsin (x / h) \\
& \zeta=(z-h \cos \beta \cos \alpha) / \cos \alpha
\end{aligned}
$$

The relations between actuated joint variables and generalized coordinates can thus be obtained in closed form. The initial values are chosen as $\alpha=\beta=0 \mathrm{rad}$, and $\zeta=70 \mathrm{~mm}$. The corresponding time-histories of the generalized coordinates, velocities, and accelerations, as well as moments and forces acting on the MP are calculated, as plotted in Figs. 4-6, while their counterparts of the actuated joints are shown in Figs. 7-10.

\section{Conclusions}

A modeling methodology applicable to redundantly-actuated systems, based on the NOC, was introduced. The method described was then applied to a redundantly-actuated PKM. The underdetermined actuator-torque array is optimized via 


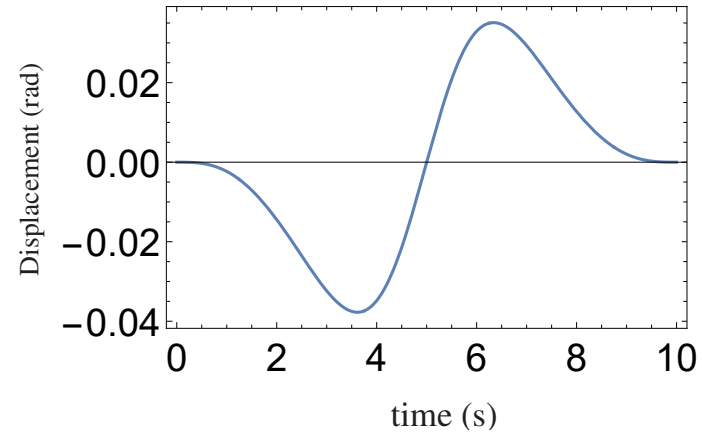

(a)

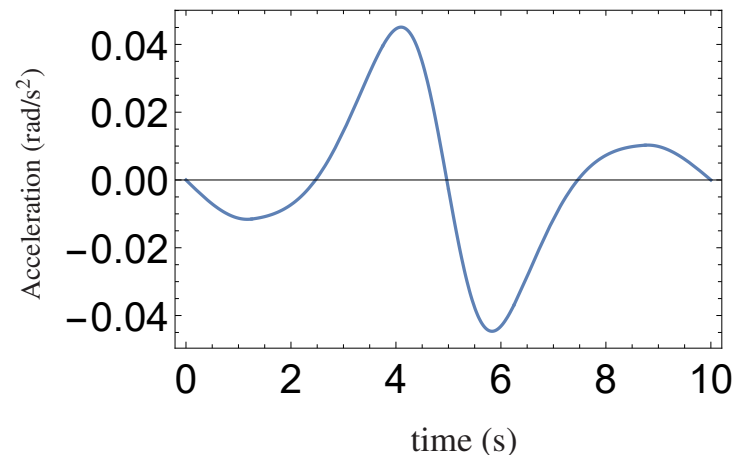

(c)

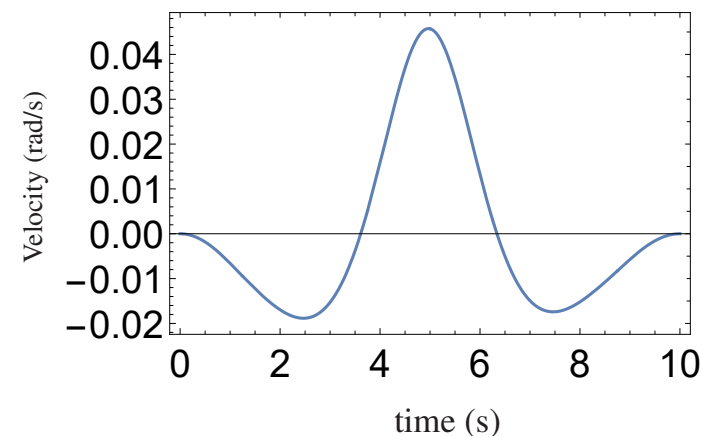

(b)

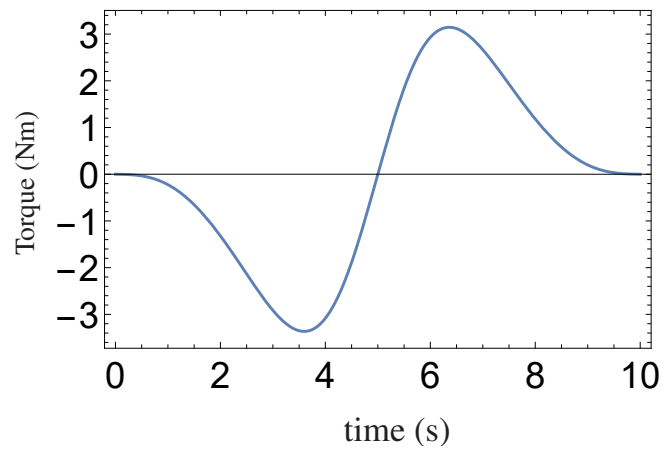

(d)

Fig. 4: Displacement, velocity, acceleration, and torque of generalized coordinate $\alpha$

Euclidean-norm minimization. A robust methodology was introduced to compute the minimum-norm actuator-torque array of the PKM under study. It is pointed out, however, that internal forces can be generated under actuation redundancy [30], thereby inducing additional loads on the structure. Hence, great care should be taken upon application of control schemes to redundantly-actuated systems, as unavoidable geometric errors of both the geometry and the control system can produce unacceptably high internal forces. To cope with this downside, a robust approach to the dimensioning of the various links is recommended as an issue worth further research.

\section{Acknowledgments}

The first author would like, first, to acknowledge the China Scholarship Council (CSC) (No. 201708330573) and the Department of Education of Zhejiang Province (Y201430657) for their financial support. Then, the same author acknowledges the use of the research facilities at the Centre for Intelligent Machines at McGill University. The work was supported by: the National Science Foundation of China (NSFC) under Grant Nos. 51525504 and U1713202; and Natural Science Foundation of Zhejiang Province under Grant LZ14E050005. The second author acknowledges the support of the Natural Sciences and Engineering Research Council of Canada (NSERC) through its Postdoctoral Fellowship Program. The third author acknowledges NSERC's support through Grant No. 4532-2010. 


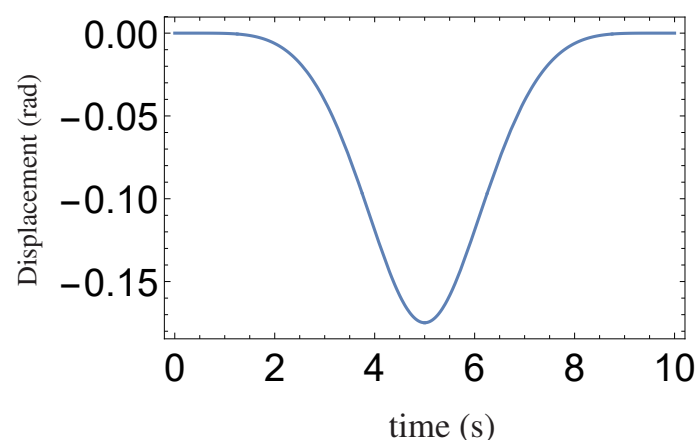

(a)

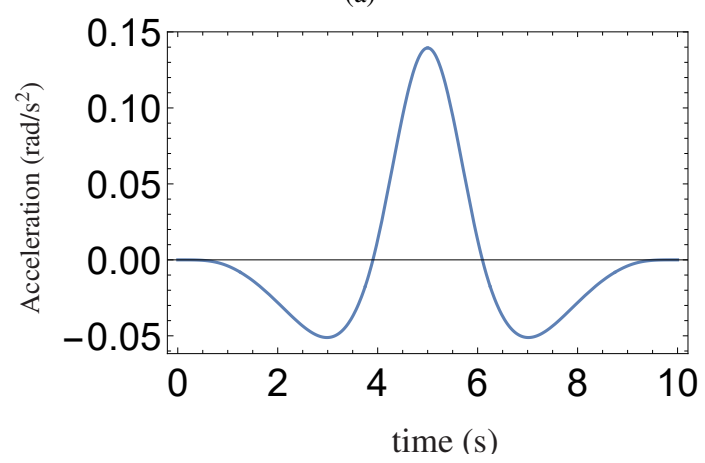

(c)

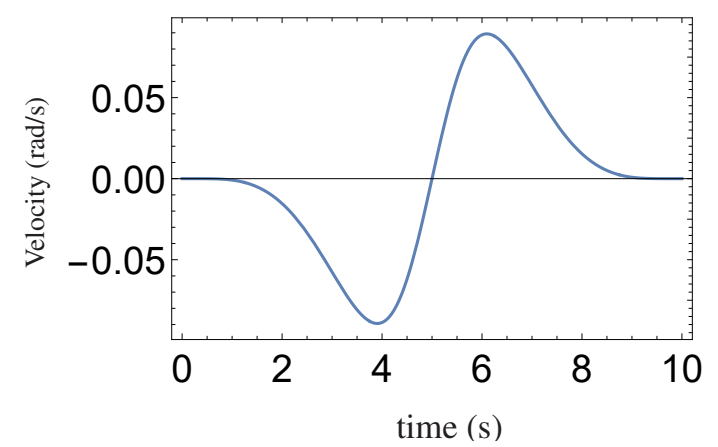

(b)

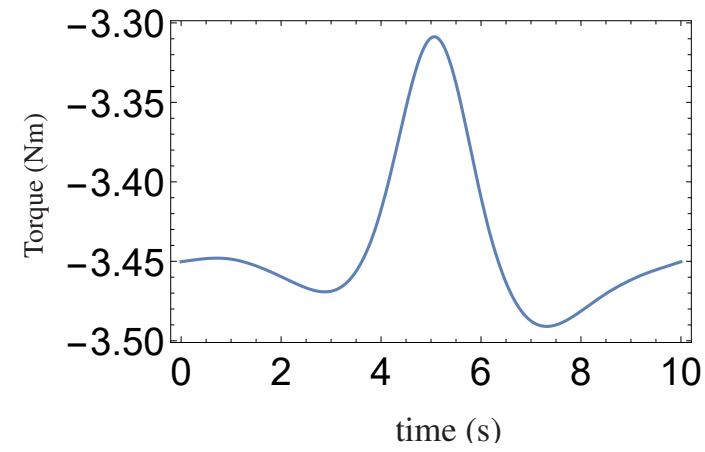

(d)

Fig. 5: Displacement, velocity, acceleration, and torque of generalized coordinate $\beta$

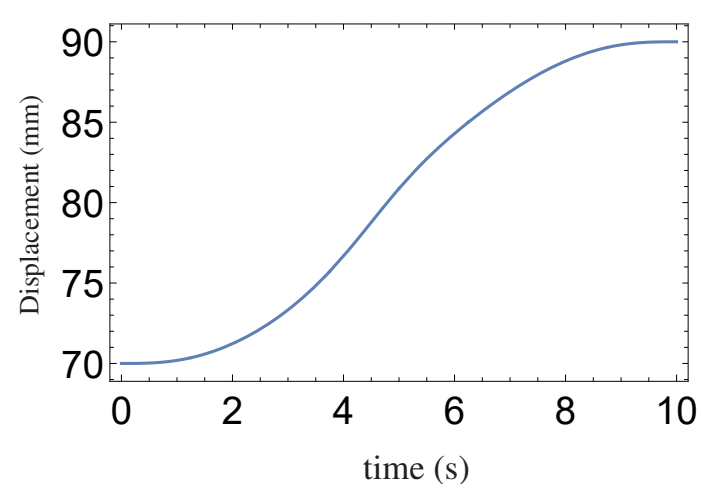

(a)

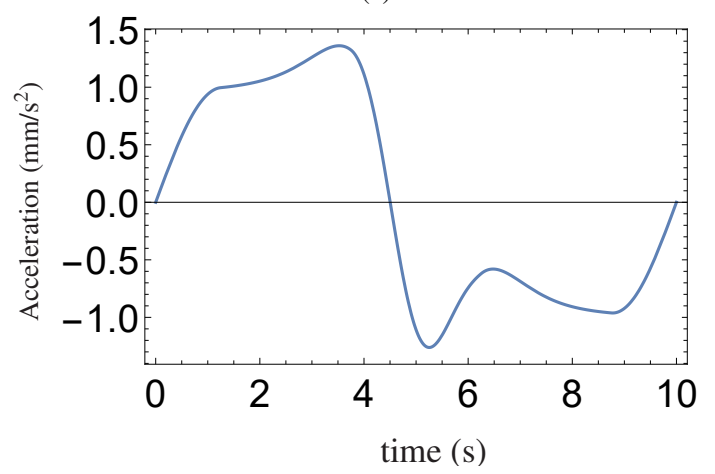

(c)

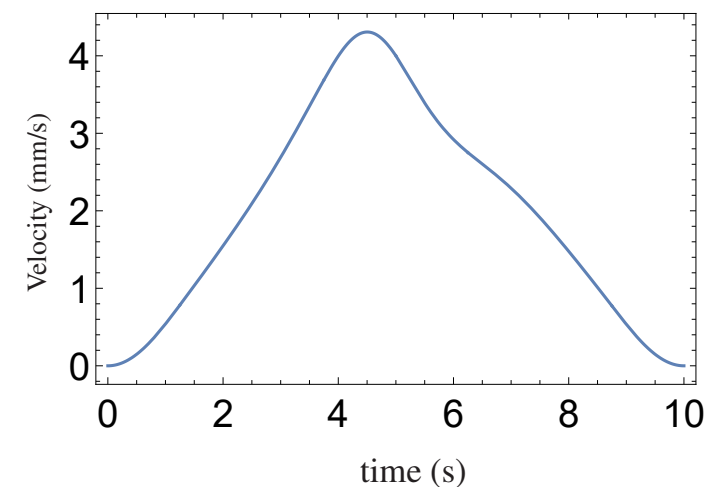

(b)

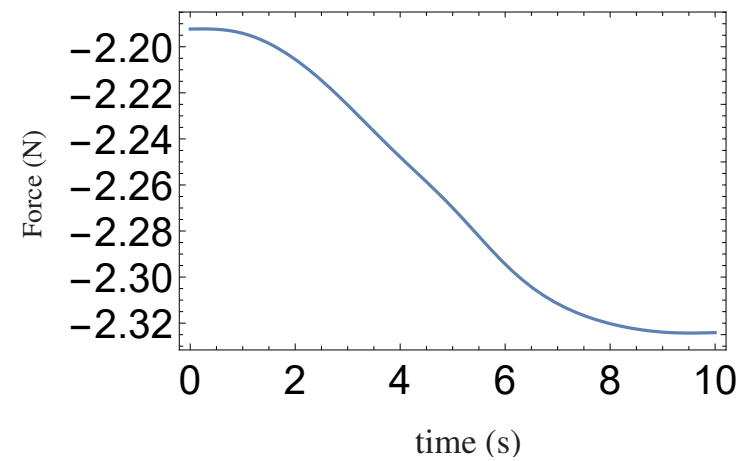

(d)

Fig. 6: Displacement, velocity, acceleration, and force of generalized coordinate $\zeta$ 


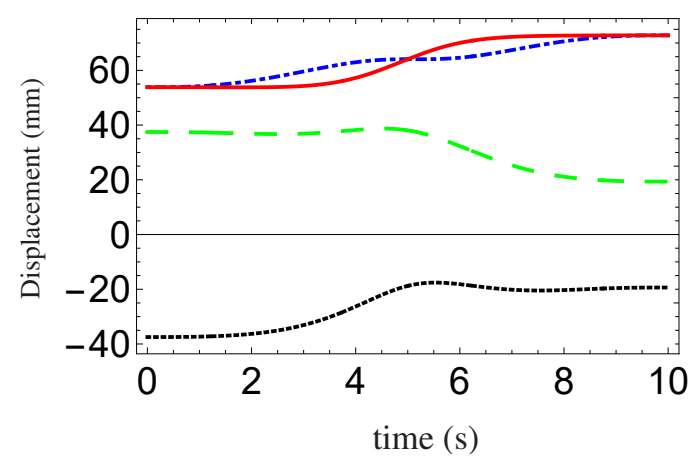

Fig. 7: Actuated-joint displacements

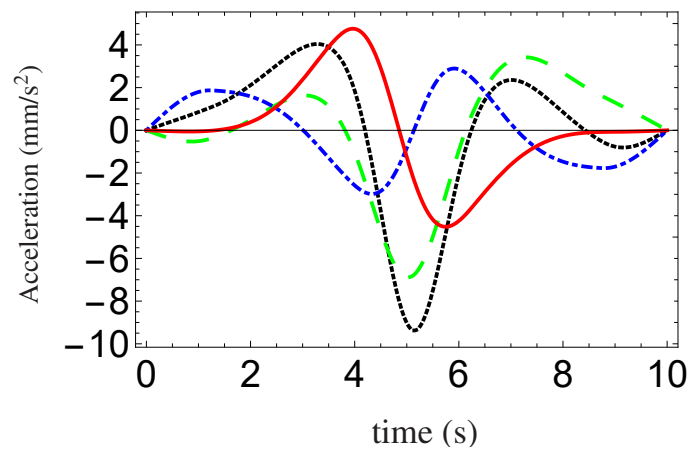

Fig. 9: Actuated-joint accelerations

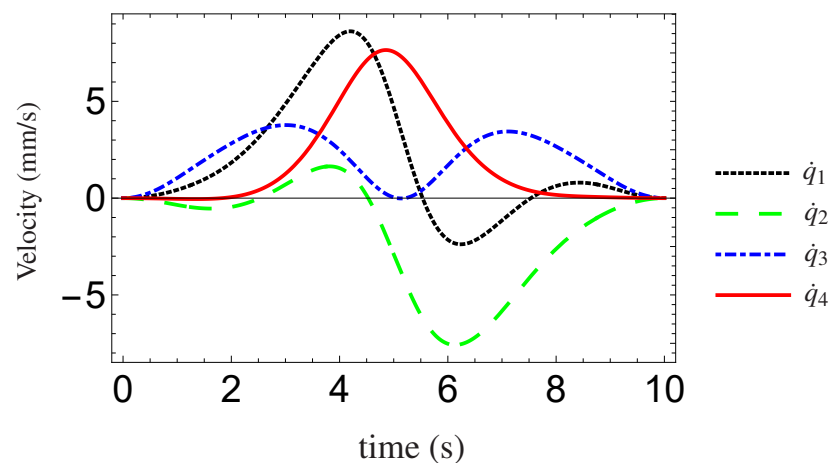

Fig. 8: Actuated-joint velocities

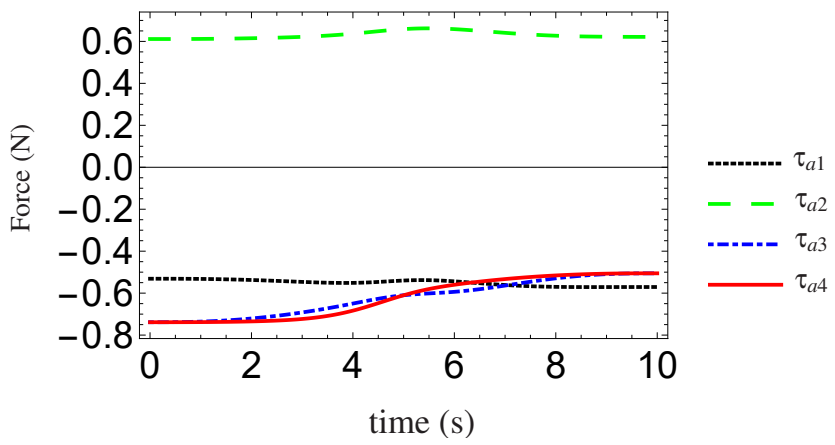

Fig. 10: Actuator-forces 


\section{Appendix A}

The seven twist-shaping matrices are:

$$
\begin{aligned}
& \mathbf{T}_{1}=\left[\begin{array}{cccccc}
0 & 0 & 0 & 0 & 0 & 0 \\
0 & 0 & 0 & -k_{11} g_{12} / g_{11}-k_{12} & 0 & 0 \\
0 & 0 & 0 & g_{12} / g_{11} & 0 & 0
\end{array}\right]^{T} \\
& \mathbf{T}_{2}=\left[\begin{array}{cccccc}
0 & 0 & 0 & 0 & 0 & 0 \\
0 & 0 & 0 & k_{22}-k_{21} g_{22} / g_{21} & 0 & 0 \\
0 & 0 & 0 & -g_{22} / g_{21} & 0 & 0
\end{array}\right]^{T}
\end{aligned}
$$

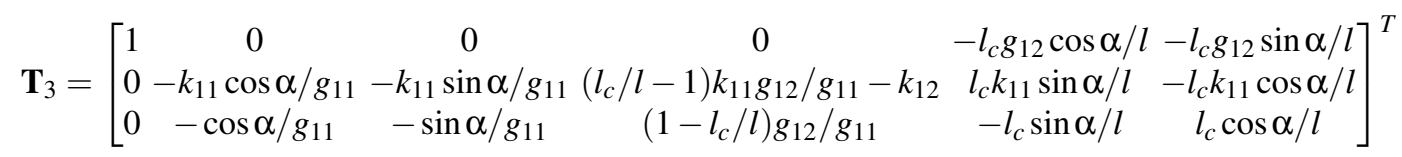

$$
\begin{aligned}
& \mathbf{T}_{4}=\left[\begin{array}{cccccc}
1 & 0 & 0 & 0 & -l_{c} g_{22} \cos \alpha / l & -l_{c} g_{22} \sin \alpha / l \\
0-k_{21} \cos \alpha / g_{21} & -k_{21} \sin \alpha / g_{21} & -\left(1+l_{c} / l\right) k_{21} g_{22} / g_{21}+k_{22} & -l_{c} k_{21} \sin \alpha / l & l_{c} k_{21} \cos \alpha / l \\
0 & -\cos \alpha / g_{21} & -\sin \alpha / g_{21} & -\left(1+l_{c} / l\right) g_{22} / g_{21} & -l_{c} \sin \alpha / l & l_{c} \cos \alpha / l
\end{array}\right]^{T} \\
& \mathbf{T}_{5}=\left[\begin{array}{cccccc}
\left(g_{31} k_{32}-g_{32} k_{31}\right) / g_{33} & 0 & 0 & 0 & k_{33}\left(g_{31} k_{32}-g_{32} k_{31}\right) / g_{33}-k_{32} & k_{34}\left(g_{32} k_{31}-g_{31} k_{32}\right) / g_{33}-k_{31} \\
0 & 0 & 0 & 0 & 0 & 0 \\
\left(g_{31} \sin \alpha+g_{32} \cos \alpha\right) / g_{33} & 0 & 0 & 0 & k_{33}\left(g_{31} \sin \alpha+g_{32} \cos \alpha\right) / g_{33}-\sin \alpha \cos \alpha-k_{34}\left(g_{31} \sin \alpha+g_{32} \cos \alpha\right) / g_{33}
\end{array}\right]^{T} \\
& \mathbf{T}_{6}=\left[\begin{array}{cccccc}
\left(g_{42} k_{41}+g_{41} k_{42}\right) / g_{43} & 0 & 0 & 0 & k_{43}\left(g_{41} k_{42}+g_{42} k_{41}\right) / g_{43}-k_{42} & -k_{44}\left(g_{41} k_{42}+g_{42} k_{41}\right) / g_{43}+k_{41} \\
0 & 0 & 0 & 0 & 0 & 0 \\
\left(g_{42} \cos \alpha+g_{41} \sin \alpha\right) / g_{43} & 0 & 0 & 0 & k_{43}\left(g_{41} \sin \alpha+g_{42} \cos \alpha\right) / g_{43}-\sin \alpha \cos \alpha-k_{44}\left(g_{41} \sin \alpha+g_{42} \cos \alpha\right) / g_{43}
\end{array}\right]^{T} \\
& \mathbf{T}_{7}=\left[\begin{array}{cccccc}
1 & 0 & 0 & 0 & 0 & 0 \\
0 & \cos \alpha \sin \alpha & 0 & 0 & 0 \\
0 & 0 & 0 & \sin \beta-\sin \alpha \cos \beta & \cos \alpha \cos \beta
\end{array}\right]^{T}
\end{aligned}
$$

with the definitions below:

$$
\begin{aligned}
& k_{11}=f \cos \beta-e \sin \beta, \quad k_{12}=e \cos \beta+f \sin \beta, \quad k_{21}=f \cos \beta+e \sin \beta, \quad k_{22}=f \sin \beta-e \cos \beta \\
& k_{31}=\zeta \sin \alpha+f \cos \alpha, \quad k_{32}=\zeta \cos \alpha-f \sin \alpha, \quad k_{33}=q_{c} g_{31} / \sqrt{g_{31}^{2}+g_{32}^{2}}, \quad k_{34}=q_{c} g_{32} / \sqrt{g_{31}^{2}+g_{32}^{2}} \\
& k_{41}=f \cos \alpha-\zeta \sin \alpha, \quad k_{42}=f \sin \alpha+\zeta \cos \alpha, \quad k_{43}=q_{c} g_{41} / \sqrt{g_{41}^{2}+g_{42}^{2}}, \quad k_{44}=q_{c} g_{42} / \sqrt{g_{41}^{2}+g_{42}^{2}} \\
& g_{11}=\sqrt{l^{2}-(\zeta-f \sin \beta-e \cos \beta)^{2}}, \quad g_{12}=\zeta-f \sin \beta-e \cos \beta \\
& g_{21}=\sqrt{l^{2}-(\zeta+f \sin \beta-e \cos \beta)^{2}}, \quad g_{22}=\zeta+f \sin \beta-e \cos \beta \\
& g_{31}=\zeta \cos \alpha-f \sin \alpha-d, \quad g_{32}=l_{3}-f \cos \alpha-\zeta \sin \alpha, \quad g_{33}=g_{31}^{2}+g_{32}^{2} \\
& g_{41}=\zeta \cos \alpha+f \sin \alpha-d, \quad g_{42}=-l_{3}+f \cos \alpha-\zeta \sin \alpha, \quad g_{43}=g_{41}^{2}+g_{42}^{2}
\end{aligned}
$$




\section{Appendix B}

The expression of the modified trapezoidal trajectory:

$$
\phi(t)= \begin{cases}\frac{2 \pi}{2+\pi}\left[\frac{2 t}{T}-\frac{1}{2 \pi} \sin \left(\frac{4 \pi t}{T}\right)\right], & 0 \leq t<\frac{T}{8} \\ \frac{2 \pi}{2+\pi}\left[\frac{1}{4}-\frac{1}{2 \pi}+\frac{2}{T}\left(t-\frac{T}{8}\right)+\frac{4 \pi}{T^{2}}\left(t-\frac{T}{8}\right)^{2}\right], & \frac{T}{8} \leq t<\frac{3}{8} T \\ \frac{2 \pi}{2+\pi}\left[-\frac{\pi}{2}+2(1+\pi) \frac{t}{T}-\frac{1}{2 \pi} \sin \left(\frac{4 \pi}{T}\left(t-\frac{T}{4}\right)\right)\right], & \frac{3}{8} T \leq t<\frac{T}{2} \\ 2 \pi+\frac{2 \pi}{2+\pi}\left[\frac{\pi}{2}+2(1+\pi) \frac{t-T}{T}-\frac{1}{2 \pi} \sin \left(\frac{4 \pi}{T}\left(t-\frac{3 T}{4}\right)\right)\right], & \frac{T}{2} \leq t<\frac{5}{8} T \\ 2 \pi+\frac{2 \pi}{2+\pi}\left[-\frac{1}{4}+\frac{1}{2 \pi}+\frac{2}{T}\left(t-\frac{7 T}{8}\right)-\frac{4 \pi}{T^{2}}\left(t-\frac{7 T}{8}\right)^{2}\right], & \frac{5}{8} T \leq t<\frac{7}{8} T \\ 2 \pi+\frac{2 \pi}{2+\pi}\left[\frac{2(t-T)}{T}-\frac{1}{2 \pi} \sin \left(\frac{4 \pi}{T}(t-T)\right)\right], & \frac{7}{8} T \leq t \leq T\end{cases}
$$

\section{References}

[1] Kane, T. R., and Levinson, D. A., 1983. "The use of Kane's dynamical equations in robotics". The International Journal of Robotics Research, 2(3), pp. 3-21.

[2] Angeles, J., and Lee, S. K., 1989. "The modelling of holonomic mechanical systems using a natural orthogonal complement". Transactions of the Canadian Society for Mechanical Engineering, 13(4), pp. 81-89.

[3] Khalil, W., and Ibrahim, O., 2007. "General solution for the dynamic modeling of parallel robots". Journal of Intelligent and Robotic Systems, 49(1), pp. 19-37.

[4] Codourey, A., and Burdet, E., 1997. "A body-oriented method for finding a linear form of the dynamic equation of fully parallel robots". In Proceedings of the IEEE International Conference on Robotics and Automation, Albuquerque, NM, April 25-25, 1997, Vol. 2, IEEE, pp. 1612-1618.

[5] Wang, J., and Gosselin, C. M., 1998. "A new approach for the dynamic analysis of parallel manipulators". Multibody System Dynamics, 2(3), pp. 317-334.

[6] Tsai, L.-W., 2000. "Solving the inverse dynamics of a stewart-gough manipulator by the principle of virtual work". Journal of Mechanical Design, 122(1), pp. 3-9.

[7] Cheng, H., Yiu, Y.-K., and Li, Z., 2003. "Dynamics and control of redundantly actuated parallel manipulators". IEEE/ASME Transactions on Mechatronics, 8(4), pp. 483-491.

[8] Miller, K., 2004. "Optimal design and modeling of spatial parallel manipulators". The International Journal of Robotics Research, 23(2), pp. 127-140.

[9] Mladenova, C. D., 1999. "Applications of lie group theory to the modeling and control of multibody systems". Multibody System Dynamics, 3(4), pp. 367-380.

[10] Wang, J., Gosselin, C. M., and Cheng, L., 2002. "Modeling and simulation of robotic systems with closed kinematic chains using the virtual spring approach". Multibody System Dynamics, 7(2), pp. 145-170.

[11] Gallardo, J., Rico, J., Frisoli, A., Checcacci, D., and Bergamasco, M., 2003. "Dynamics of parallel manipulators by means of screw theory". Mechanism and Machine Theory, 38(11), pp. 1113-1131.

[12] Staicu, S., and Zhang, D., 2008. "A novel dynamic modelling approach for parallel mechanisms analysis". Robotics and Computer-Integrated Manufacturing, 24(1), pp. 167-172.

[13] Mendes Lopes, A., and Almeida, F., 2009. "The generalized momentum approach to the dynamic modeling of a 6-dof parallel manipulator". Multibody System Dynamics, 21(2), pp. 123-146.

[14] Huston, R., and Passerello, C., 1974. "On constraint equations-a new approach". Journal of Applied Mechanics, 41(4), pp. 1130-1131.

[15] Hemami, H., and Weimer, F., 1981. "Modeling of nonholonomic dynamic systems with applications". Journal of Applied Mechanics, 48(1), pp. 177-182.

[16] Wehage, R., and Haug, E., 1982. "Generalized coordinate partitioning for dimension reduction in analysis of constrained dynamic systems". Journal of mechanical design, 104(1), pp. 247-255.

[17] Kamman, J. W., and Huston, R., 1984. "Dynamics of constrained multibody systems". Journal of Applied Mechanics, 51(4), pp. 899-903.

[18] Lipkin, H., and Duffy, J., 1985. "On the geometry of orthogonal and reciprocal screws". In Theory and Practice of Robots and Manipulators. Springer, pp. 47-55.

[19] Angeles, J., 2014. Fundamentals of Robotic Mechanical Systems Theory, Methods, and Algorithms. Springer.

[20] Gosselin, C. M., and Schreiber, L.-T., 2018. "Redundancy in parallel mechanisms: a review". Applied Mechanics Reviews, 70(1), p. 010802.

[21] Wang, Y., Belzile, B., Angeles, J., and Li, Q., 2019. "Kinematic analysis and optimum design of a novel 2pur-2rpu parallel robot". Mechanism and Machine Theory, 139, pp. 407-423. 
[22] Constantinescu, D., Chau, I., DiMaio, S. P., Filipozzi, L., Salcudean, S. E., and Ghassemi, F., 2000. "Haptic rendering of planar rigid-body motion using a redundant parallel mechanism". In Proceedings of the IEEE International Conference on Robotics and Automation, San Francisco, CA, April 24-28, 2000, Vol. 3, IEEE, pp. 2440-2445.

[23] Wu, J., Wang, J., Wang, L., and Li, T., 2009. "Dynamics and control of a planar 3-dof parallel manipulator with actuation redundancy". Mechanism and Machine Theory, 44(4), pp. 835-849.

[24] Xu, L., Li, Q., Zhang, N., and Chen, Q., 2017. "Mobility, kinematic analysis, and dimensional optimization of new three-degrees-of-freedom parallel manipulator with actuation redundancy". Journal of Mechanisms and Robotics, 9(4), p. 041008.

[25] Wang, C., Fang, Y., Guo, S., and Chen, Y., 2013. "Design and kinematical performance analysis of a 3-rus/rrr redundantly actuated parallel mechanism for ankle rehabilitation". Journal of Mechanisms and Robotics, 5(4), p. 041003.

[26] Golub, G. H., and Van Loan, C. F., 2012. Matrix Computations, Vol. 3. JHU Press.

[27] Wang, Y., Belzile, B., Angeles, J., and Li, Q., 2019. "On the modeling of redundantly-actuated mechanical systems". In Proceedings of the 9th ECCOMAS Thematic Conference on Multibody Dynamics, Duisburg, Germany, July 15-18, 2019, Springer, pp. 172-179.

[28] Mises, R., 1924. "Motorrechnung, ein neues hilfsmittel der mechanik". ZAMM-Journal of Applied Mathematics and Mechanics/Zeitschrift für Angewandte Mathematik und Mechanik, 4(2), pp. 155-181.

[29] Ghorbel, F., Chételat, O., and Longchamp, R., 1994. "A reduced model for constrained rigid bodies with application to parallel robots". In Proceedings of the IFAC Symposium on Robot Control, Capri, Italy, September 19-21, 1994, Citeseer, pp. 57-62.

[30] Müller, A., 2005. "Internal preload control of redundantly actuated parallel manipulators-its application to backlash avoiding control". IEEE Transactions on Robotics, 21(4), pp. 668-677.

[31] Tian, Q., Flores, P., and Lankarani, H. M., 2018. "A comprehensive survey of the analytical, numerical and experimental methodologies for dynamics of multibody mechanical systems with clearance or imperfect joints". Mechanism and Machine Theory, 122, pp. 1-57.

[32] Ben-Israel, A., and Greville, T. N., 2003. Generalized Inverses: Theory and Applications, Vol. 15. Springer Science \& Business Media.

[33] Siciliano, B., 1999. "The Tricept robot: Inverse kinematics, manipulability analysis and closed-loop direct kinematics algorithm". Robotica, 17(4), pp. 437-445.

[34] Wahl, J., 2002. Articulated tool head: US Patent, 6431802.

[35] Zoppi, M., Zlatanov, D., and Molfino, R., 2010. "Kinematics analysis of the Exechon tripod". In ASME 2010 International Design Engineering Technical Conferences and Computers and Information in Engineering Conference, American Society of Mechanical Engineers, pp. 1381-1388.

[36] Bi, Z. M., and Jin, Y., 2011. "Kinematic modeling of Exechon parallel kinematic machine". Robotics and ComputerIntegrated Manufacturing, 27(1), pp. 186-193.

[37] Ni, Y., Zhang, B., Sun, Y., and Zhang, Y., 2016. "Accuracy analysis and design of A3 parallel spindle head". Chinese Journal of Mechanical Engineering, 29(2), pp. 239-249.

[38] Eskandary, P. K., and Angeles, J., 2018. "The dynamics of a parallel schönflies-motion generator". Mechanism and Machine Theory, 119, pp. 119-129.

[39] Wang, L., Xu, H., and Guan, L., 2017. "Kinematics and inverse dynamics analysis for a novel 3-puu parallel mechanism”. Robotica, 35(10), pp. 2018-2035.

[40] Biagiotti, L., and Melchiorri, C., 2008. Trajectory Planning for Automatic Machines and Robots. Springer Science \& Business Media. 


\section{List of Tables}

$1 \quad$ Robot Geometric Data $\ldots \ldots \ldots \ldots \ldots \ldots \ldots \ldots$

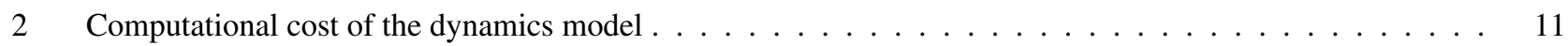




\section{List of Figures}

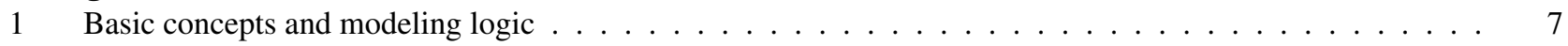

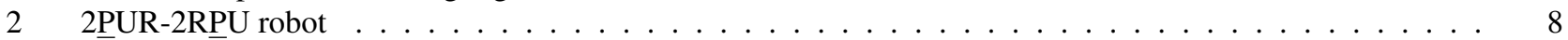

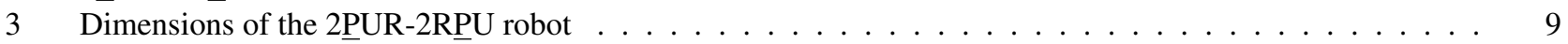

4 Displacement, velocity, acceleration, and torque of generalized coordinate $\alpha \ldots \ldots \ldots \ldots$

5 Displacement, velocity, acceleration, and torque of generalized coordinate $\beta \ldots \ldots \ldots \ldots \ldots$

6 Displacement, velocity, acceleration, and force of generalized coordinate $\zeta \ldots \ldots \ldots \ldots \ldots$

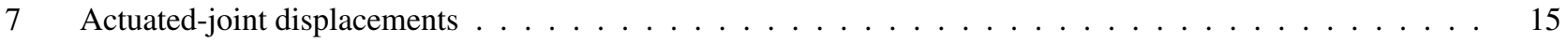

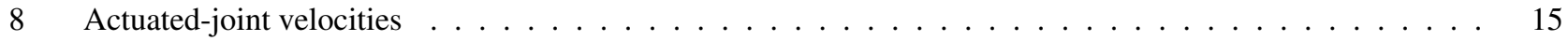

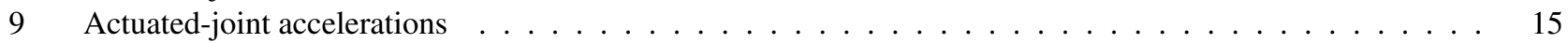

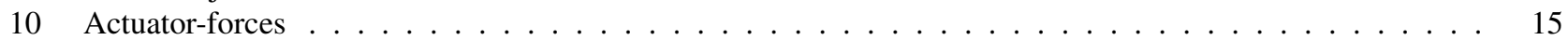

